\title{
Immune response of standard and delayed hepatitis B vaccination schedules among medical students - a cross sectional comparative study
}

\author{
Ansiya A* and M P Saraswathy \\ Assistant Professor, Dept. of Microbiology, CRRI, ESIC Medical College \& PGIMSR, KK Nagar, \\ Chennai
}

\begin{abstract}
Context: Health care professionals including medical students face a high risk for Hepatitis B transmission. Hepatitis B vaccination is routinely administered to health care providers and medical students. However due to the rigors of medical training, medical students miss doses of the vaccine. There is scant research on immune response following delayed immunization schedule. Aims: To compare immune response as measured by anti-HBsAg antibody levels in medical students who followed delayed and standard vaccination schedule of Hepatitis B vaccination. Settings and designs: A cross sectional comparative study was conducted among randomly selected 50 medical students of a medical college in Chennai. Methods and Materials: Data on demographics, Hepatitis B immunization details and other covariants were collected. A two step immunoassay, using Chemiluminescent Microparticle Immunoassay (CMIA) technology was used for the quantitative determination of anti-HBs in human serum and plasma. The collected data was analyzed using statistical software IBM SPSS Statistical Package version 21. Results: Of the students, 36 (72\%) were females and 14 (28\%) were males. Among the participants who followed standard immunization schedule without delay, $6.3 \%$ had poor immune response, and $12.5 \%$ were hypo-responsive. Among the participants who followed delayed schedule, $2.9 \%$ were non-responsive and $5.9 \%$ were hypo-responsive. After adjusting for age, gender, BMI and delay in schedule, it was found that greater gap between dose 1 and dose $2(p=0.001)$ and gap between dose 2 and dose $3(p=0.004)$ significantly influenced the anti-HBsAg antibody titres. Conclusion: It is seen that a delayed or altered vaccination schedule did not seriously affect the immune response among medical students. Therefore, the
\end{abstract}

*Author for Correspondence. E-mail: ansiyaabbasali@gmail.com www. ijamicro.com 
focus should be on completing the immunization schedule of Hepatitis B at the best available opportunity.

K eywords: hepatitis B Vaccination, Immunization schedule, Anti-HBsAg titre, Immune response, Delayed schedule.

\section{Introduction}

Hepatitis B virus infection is a serious problem world over. [1] More than 400 million people are infected with hepatitis B virus worldwide. This virus is a major cause of liver cirrhosis, chronic hepatitis and hepatocellular carcinoma $[2,3]$.

Health care workers are at a high risk of HBV infection as they are more prone to occupational exposure to blood, and the incidence of this infection among them has been estimated to be 2-4 times the level in the general population [4] Health care workers have been reported to have the highest occupational risk of HBV infection during their health professional training [5]. As a part of occupational safety measures, medical students along with all other health care workers are supposed to be vaccinated against HBV [6,7].Vaccination is a cost-effective way to prevent HBV infection, and in turn, to reduce the incidence of acute and chronic hepatitis B, hepatic cirrhosis, and hepatocellular carcinoma [8].

Worldwide, the standard hepatitis B vaccination schedule for adults consists of three doses administered on a $0-1-6$ month schedule, which typically results in at least $85 \%$ seroprotection in target groups [9] in which there should be minimal dosing interval of 4 weeks between first and second doses, 8 week between second and third doses, and 16 weeks between 1st and third doses [10]. Post vaccination, anti $\mathrm{HBsAg}$ antibody titre level of $\geq 10 \mathrm{mIU} / \mathrm{ml}$ is considered seroprotective [11]; $<10 \mathrm{mIU} / \mathrm{ml}$ as non responsiveness to $\mathrm{HBV}$ vaccination; between 10 and 100 $\mathrm{mIU} / \mathrm{ml}$ as hypo responsiveness to $\mathrm{HBV}$ vaccination and levels $>100 \mathrm{mIU} / \mathrm{ml}$ are taken as a high level of immunity. [12]

It has been noticed that, due to stress, work load, large syllabus and tight schedule of postings, many medical students miss a dose of vaccination schedule and many of them complete the 3 doses in alternatively delayed manner. This research study will analyze the immune response among the medical students in a medical college of Chennai, as measured by anti HBsAg antibody level and will study various factors contributing to this variation of immune responses among them and to know how much delayed and standard schedules of vaccination will influence antibody levels.

\section{M aterials and M ethods}

Study was started after getting approval of institutional ethics committee on13/7/16 and it's a ICMR funded STS project, under the Department of Microbiology, ESIC MC AND PGIMSR Chennai. 
A cross sectional comparative study was conducted from August 2016 to October 2016 among randomly selected 50 medical students of a medical college in Chennai. Samples were selected by simple random sampling technique. Unique code numbers were assigned to all subjects to preserve confidentiality. Samples were obtained from all eligible students who provided written informed consent to participate.

A self administered data collection proforma included questions on demographics, including age, gender, height and weight; Hepatitis B immunization details (birth and child hood immunization, immunization at the time of college entry, number of doses, normal or delayed, reason for delayed); Details on covariant that may cause variation of immune responses (jaundice, blood transfusions, blood and body fluid contacts, habit of smoking etc). A two step immunoassay, using chemiluminescent microparticle immunoassay (CMIA) technology was used for the quantitative determination of anti-HBs in human serum and plasma. The collected data was entered in MS Excel spreadsheet. The data validity was verified as the researcher entered the data. The data was imported to IBM SPSS Statistical Package version 21 for further analysis. Descriptive statistics was performed to study the characteristics of study participants. Anti HBsAg titre levels were statistically analysed, $\mathrm{P}$ value lesser than 0.05 was considered as statistically significant.

\section{Results}

In this study we enrolled 50 medical students in the clinical year (II and III year), among which $36(72 \%)$ were females and 14(28\%) were males, aged between 19 to 22 years. Among 50, $6(12 \%)$ candidates were underweight, 32(64\%) participants were in normal range,9(18\%) participants were overweight and $3(6 \%)$ participants were obese. Table 1 showed the characteristics of study participants.

Table 1: Characteristics of Study Participants

\begin{tabular}{|l|l|c|c|c|}
\hline SL & Characteristic & Category & Frequency & Percentage (\%) \\
\hline 1 & AGE & 19 & 5 & 10 \\
\hline & & 20 & 16 & 32 \\
\hline & & 21 & 21 & 42 \\
\hline & & 22 & 8 & 16 \\
\hline 2 & GENDER & FEMALE & 36 & 72 \\
\hline & & MALE & 14 & 28 \\
\hline 3 & BMI & $<18.5$ & 6 & 12 \\
\hline & & $18.5-24.9$ & 32 & 64 \\
\hline & & $25-29.9$ & 9 & 18 \\
\hline & & $>30$ & 3 & 6 \\
\hline
\end{tabular}


Hepatitis B immunization profiles of participants are given in Table 2.

Table 2: Hepatitis B Immunization Profile

\begin{tabular}{|l|l|c|c|c|}
\hline SL No: & Characteristics & Category & Frequency & Percentage (\%) \\
\hline 1 & Hepatitis B Vaccination at Birth & Yes & 27 & 54 \\
\hline 2 & Hepatitis B Vaccination During Childhood & Yes & 3 & 6 \\
\hline 3 & Hepatitis B Vaccination Afterwards & Yes & 49 & 98 \\
\hline
\end{tabular}

Here, 27(54\%) participants took hepatitis vaccination at birth, rest 23(46\%) of the participants did not take hepatitis B vaccination at birth, among total enrolled 3(6\%) candidates took vaccination during childhood, rest $47(96 \%)$ candidates did not take hepatitis B vaccination during childhood; among 50 participants, 49(98\%) participants took hepatitis B vaccination afterwards and during college admissions, $1(2 \%)$ did not take hepatitis B vaccination afterwards. For studying about risk factors of hepatitis B, we included about six risk factors in the data collection proforma which was given in Table 3 .

Table 3: Hepatitis B R isk Factor Profile

\begin{tabular}{|c|l|c|c|c|}
\hline SL No: & Characteristics & Category & Frequency & Percentage (\%) \\
\hline 1 & sharing razors & Yes & 2 & 4 \\
\hline & & No & 48 & 96 \\
\hline 2 & recent dental procedures & Yes & 10 & 20 \\
\hline & & No & 40 & 80 \\
\hline 3 & history of jaundice & Yes & 6 & 12 \\
\hline & & No & 44 & 88 \\
\hline 4 & history of blood transfusion & Yes & 1 & 2 \\
\hline & & No & 49 & 98 \\
\hline 5 & habit of smoking & Yes & 0 & 0 \\
\hline & & No & 50 & 100 \\
\hline 6 & contact with blood and body fluids & Yes & 2 & 4 \\
\hline & & No & 48 & 96 \\
\hline
\end{tabular}


Figure 1 shows the histogram of Anti-HBsAg titre levels,

Fig 1 histogram of anti HBsAg titres

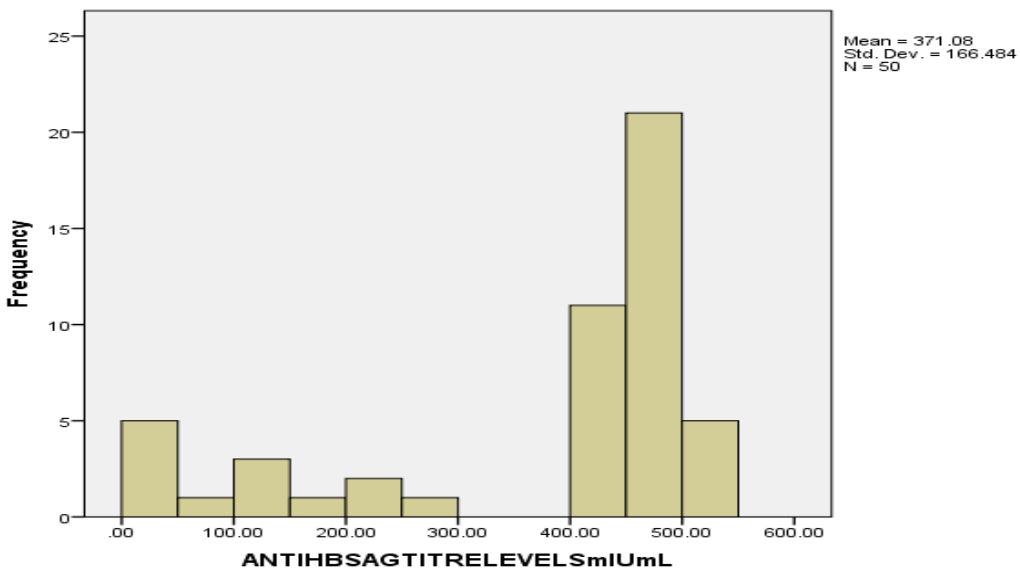

Figure 1: shows the histogram of Anti-HBsAg titre levels, we got a bimodal graph with two peaks signifying distribution of titre levels of all participants in two different ranges, 0 to $300 \mathrm{mlU} / \mathrm{mL}$ and 400 to $550 \mathrm{mlU} / \mathrm{mL}$.

In Figure 2 and Table 4, the association between immunization schedule and anti-HBsAg titre is shown.

Fig 2 Association between Immunisation Schedule And Anti H BsAg titre

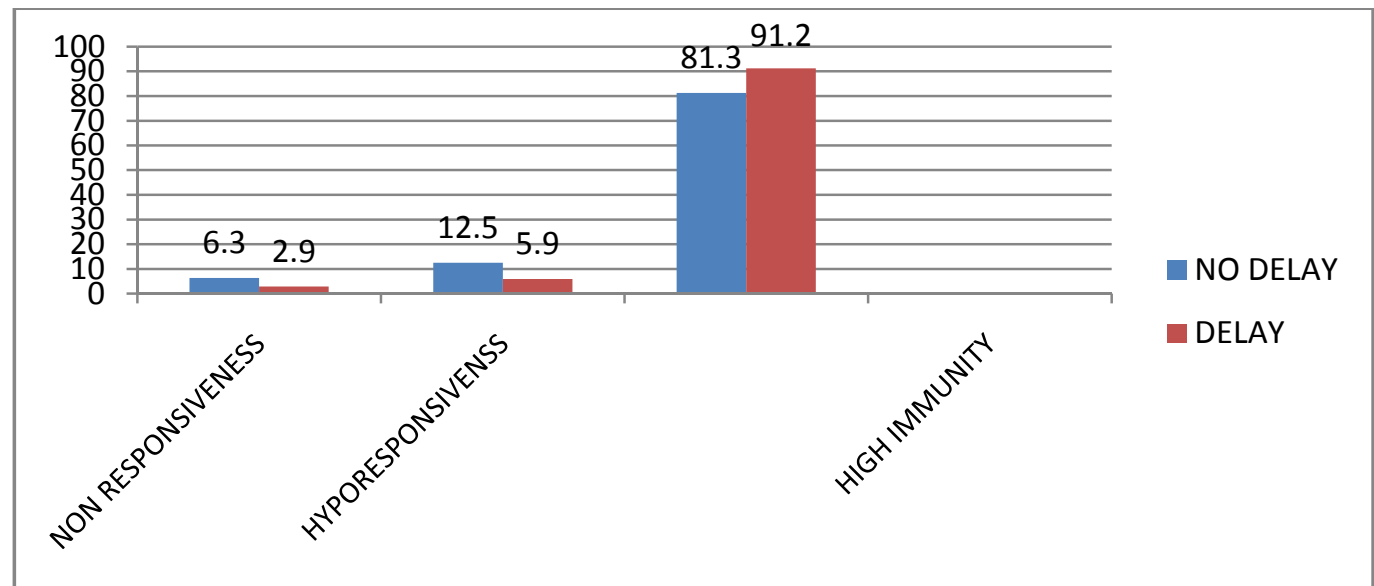

Figure 2: shows the association between immunization schedule and anti-HBsAg titre, which shows both the groups, delayed and not delayed, has a percentage of high immunity, hypo responders and non responders. 
Table 4: A ssociation between Immunisation Schedule and Anti H BsAg Titre

\begin{tabular}{|l|c|c|c|}
\hline SL No: & Non-Responsiveness & Hypo Responsiveness & High Immunity \\
\hline No Delay & 6.3 & 12.5 & 81.3 \\
\hline Delay & 2.9 & 5.9 & 91.2 \\
\hline
\end{tabular}

In figure 2 it shows, among those who followed a standard schedule $6.3 \%$ showed non responsiveness, $12.5 \%$ showed hypo responses and $81.3 \%$ have high immunity. Among those who followed a delayed schedule about $2.9 \%$ showed non-responsiveness, $5.9 \%$ showed hypo responses and $91.2 \%$ have high immunity, There is a chance that the delayed dose of vaccination acts as booster dose which will increase immunity against the antigen back to protective level,

In Table 5 the factors influencing Anti-HBs Ag titre levels are analyzed by multiple linear regressions.

Table 5: Factors Influencing A nti H bsA g titre levels, multiple L inear Regression A nalysis

\begin{tabular}{|c|l|c|c|c|}
\hline SL No & Factors Influencing Titre & $\begin{array}{c}\text { Beta } \\
\text { Coefficient }\end{array}$ & $\begin{array}{c}\text { 95\% Confidence } \\
\text { Interval }\end{array}$ & P Value \\
\hline 1 & Age & -18.488 & -71.970 to 34.94 & .488 \\
\hline 2 & Gender & -56.564 & -145.291 to 32.164 & .204 \\
\hline 3 & BMI & -5.527 & -15.732 to 4.677 & .280 \\
\hline 4 & Delayed schedule & -77.271 & -291.910 to 137.368 & .470 \\
\hline 5 & Gap between dose1 and dose 2 & -91.639 & -142.413 to -40.865 & .001 \\
\hline 6 & Gap between dose2 and dose 3 & 26.990 & 8.931 to 45.049 & .004 \\
\hline
\end{tabular}

Among various factors we considered, age $(\mathrm{p}=0.488)$, Gender $(\mathrm{p}=.204)$, BMI $(\mathrm{P}=0.280)$, Delayed schedule $(p=0.470)$ have an influence but not statistically significant. Figure 3 shows the influence of gap between dose 1 and dose 2 on Anti HBsAg titre levels,

Fig 3: Influence of G ap between Dose 1 and Dose 2 on Anti H BsAg Titre

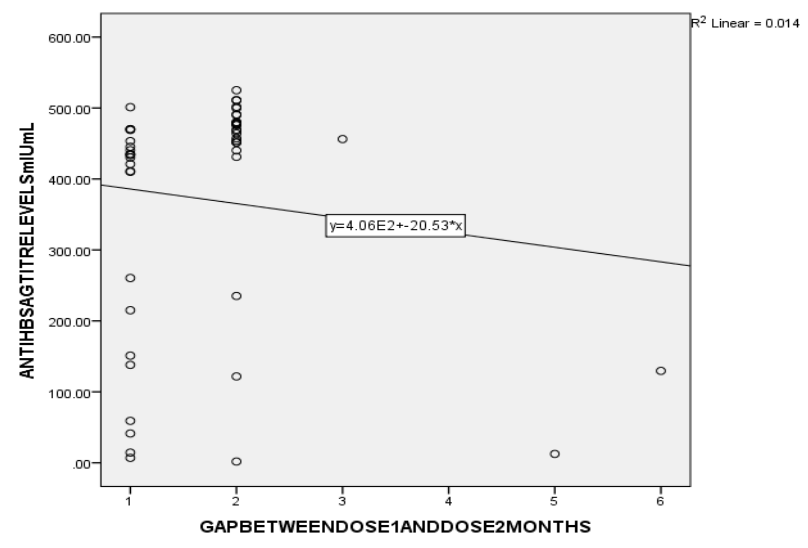


Figure 3: shows the influence of gap between dose1 and dose2 on Anti-HBsAg titre levels, Here we get a $P$ value $=0.001$ and Beta coefficient $=0.573$

\section{Fig 4 Influence of $G$ ap between Dose 2 and Dose 3 on Anti H BsA g Titre}

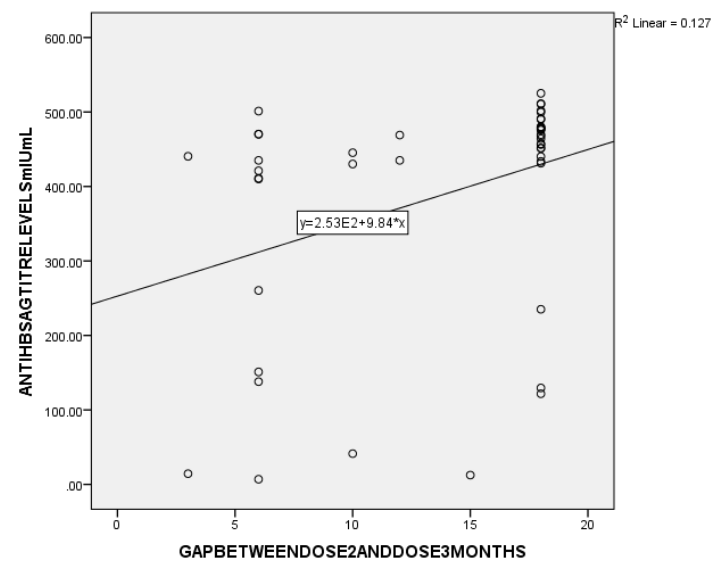

Figure 4: shows the influence of gap between dose2 and dose 3 on Anti-HBsAg titre levels, Here $P$ value $=.004$ and Beta coefficient $=.959$

\section{Discussion:}

This study was conducted to determine the anti-HBsAg antibody titres of a group of medical students in a college in Chennai, and to investigate how the standard and delayed schedule influence the variation of anti-HBsAg titre, the possible correlation between demographic features as well as details of vaccination schedule with anti-HBs antibody titer in this population.

The study population consisted of 50 medical students of age 19 to 22,36 subjects (72\%) of which were females and $14(28 \%)$ were males. In our analyses, the following percentage of people were found to have the risk factors for Hepatitis B infection: sharing razors $(4 \%)$, recent dental procedures $(20 \%)$, history of jaundice $(12 \%)$, history of blood transfusion $(2 \%)$, contact with blood and body fluids (4\%).

Analyzing the anti-HBsAg antibody levels, it showed a bimodal distribution. We were unable to find the causal attributes of this distribution and its significance. It will be taken up during future studies.

In my study, among the participants who followed standard immunization schedule without delay, $6.3 \%$ were non-responsive who are at risk of contracting hepatitis B. Even among the participants who followed standard immunization schedule, there are non-responders and hypo-responders, so it is better to check post vaccination titer levels to ensure protection. Among the participants who followed standard immunization schedule without delay, $12.5 \%$ were hypo-responsive. Their antibody levels, though protective, is not as high as the responders. Among the participants who 
followed delayed schedule, 2.9\% were non-responsive and 5.9\% were hypo-responsive. Participants found to be non responders in both the groups were recommended booster dose and they will be followed up.

According to $\mathrm{CDC}$, it is wise to check the titre levels of 1 to 2 months after completion of schedule to ensure that they have indeed responded to the vaccine because of the high risk of contracting hepatitis B virus. The non responders need post exposure prophylaxis after accidental exposure (25).

Smoking habit, obesity, genetic factors, or immune suppression may be the reason for initial lack of response $(26-29)$. Or the antibody titres of those who responded to the primary 3 doses may have gradually declined over time. It is known that an estimated $13 \%-60 \%$ of initial responders to the HBV vaccine may lose detectable anti-HBsAg antibody levels in subsequent years $(30,31)$.

On performing multiple linear regression analysis of factors influencing anti HBsAg titre levels after adjusting for age, gender, BMI and delay in schedule, it was found that gap between dose 1 and dose $2(p=0.001)$ and gap between dose 2 and dose $3(p=0.004)$ significantly correlated with anti-HBsAg levels. It is also seen that as the gap between dose 1 and dose 2 increases, the titer value decreases, whereas here when the gap between dose 2 and dose 3 increases, titre value increases as well, here the gaps show a deviation from usually followed optimal interval to produce optimal immune responses, as memory $\mathrm{T}$ cells having high proliferative potential is not formed until several weeks of first immunization and memory B cells have to go through the germinal center reaction and take several month to develop, also here the delayed $3^{\text {rd }}$ dose may act like a booster dose and act as re exposure to immunizing agent

As medical students belong to the high risk population, it is ideal to stick to the standard immunization schedule, especially the gap between the dose 1 and 2 as increasing the gap causes fall in titre levels. Delay between dose 2 and dose 3 does not cause any harm as it was observed with increase in the titre levels. Even in special circumstances, care should be taken to avoid delay between dose 1 and dose 2 .

This is one of the few studies which have looked into the delayed immunization schedule of Hepatitis B vaccination among medical students in India. However, the present study does not explain the reasons for decrease in antibody levels and its correlation with factors like immune suppression, genetic influences or smoking. Also, the study does not consider factors like site of vaccine injection, brand of injection and the dose which can produce change in the antibody levels. Random convenient sampling decreases the generalisability of the findings. Future studies must take up a large sample size and study about the reason behind variation of immune levels.

\section{Conclusion}

On comparing immune response among a group of medical students who followed standard and delayed hepatitis B immunization schedule, it is found that both the groups contain a given percentage of non responders, hypo responders and people with high immunity. After adjusting for age, gender, BMI and delay in schedule, it was found that gap between dose 1 and dose 2 
$(p=0.001)$ and gap between dose 2 and dose $3(p=0.004)$ significantly correlated with antiHBsAg anti body levels. Since there are candidates with risk of low immune responses in altered immunization schedules it is better to stick to standard universal vaccination schedule. Also it is necessary to check anti HBsAg antibody titre 1- 2 months after completion of vaccination schedule to ensure that the person has responded to vaccine or not. In future studies, we would like to look into the factors that caused variation in anti HBsAg titre levels in both standard and delayed schedules. The cause and the significance of bimodal distribution of the titre levels should also be studied.

\section{Acknowledgment}

Authors like to thank Dean, Head of the Department of Microbiology, professors of Department of Microbiology and community medicine, of ESIC Medical College and PGIMSR Chennai, who helped for the completion of the study and all the candidates who participated in the study .thanks to ICMR for funding this study.

\section{Conflicts of interest}

The authors declare no conflicts of interest.

\section{References}

1. Lule G N, et al.1989 "HBV markers (HBsAg, anti-HBc, anti-HBs) among 160 medical students at Kenyatta National Hospital”. East Afr Med J;66(5):315-318.

2. Zeeshan M, et al.2007 "Evaluation of immune response to Hepatitis B vaccine in health care workers at a tertiary care hospital in Pakistan: an observational prospective study". BMC Infect Dis; $7: 120$.

3. Varshochi M, Mahmodian R.2011 "Immunologic Response to Hepatitis B Vaccine in Health Care Workers: A Screening Program and Evaluation of Some Host-Related Factors Role." Am J Immunol;7(1):12-6.]

4. West DJ.1984. "The risk of hepatitis B infection among health professionals in the United States: a review”. Am J Med Sc; 287(2):26-33.

5. Prüss-Üstün A,2005. "Estimation of the global burden of disease attributable to contaminated sharps injuries among health-care workers". Am J Ind Med;48(6):482-490.

6. Mahoney FJ.1999 "Update on diagnosis, management and prevention of hepatitis B virus infection”. Clin Microbiol Rev;12(2):351-366.

7. U.S. Public Health Service.2001. "Updated U.S: public health service guidelines for the management of occupational exposures to $\mathrm{HBV}, \mathrm{HCV}$, and $\mathrm{HIV}$ and recommendations for postexposure prophylaxis". MMWR Recomm Rep;50(RR-11):1-52.

8. World Health Organization. Department of Communicable Disease Surveillance and Response.2002. Hepatitis B; 2:1-76. 
9. Asli AA, et al.2011. "Vaccination against hepatitis B among prisoners in Iran: accelerated vs. classic vaccination. Health Policy"; 100(2-3):297-304.

10. Centre for Disease Control and Prevention Epidemiology and prevention of vaccine- Preventable disease.2007.10th edn. Washington, DC: public health foundation.

11. Chaves SS, et al.2012 "Persistence of long-term immunity to hepatitis B among adolescents immunized at birth". Vaccine;30:1644-9.

12. Zuckerman, et al.1997. "Immune response to a new hepatitis B vaccine in healthcare workers who had not responded to standard vaccine: randomised double blind dose-response study". BMJ; 314: 329-333

13. Cárdenas-Perea ME, et al.2016 "Mexico. human vaccines and immunotherapeutics"; 12(7):185762.

14. Batra V,et al.2015.Hepatitis B immunization in healthcare workers. Annals of Gastroenterology: quarterly publications of Hellenics Society of gastroenterology. Apr-Jun;28(2):276-280.

15. Papagiannis D, et al.2016, Greece. International Journal of environmental research \& public health. Mar 15;13(3).

16. Ibrahim N, Idris A.2014 "Hepatitis B Awareness among Medical Students and Their Vaccination Status at Syrian Private University". Hepatitis Research and Treatment.;2014: DOI:10.1155/2014/131920

17. JiglW, SchmidtM, Deinhardt F. "Vaccination against hepatitis B: comparison of three different vaccination schedule".J infect Dis.

18. Jin H,et al.2015,"Comparison of Accelerated and Standard Hepatitis B Vaccination Schedules in High-Risk Healthy Adults: A Meta-Analysis of Randomized Controlled Trials". PLoS One. Jul $21 ; 10(7)$.

19. Keyghobad Ghadiri, 2012. "Comparison of the accelerated and standard vaccination schedules against hepatitis B in healthcare workers. Journal of research In medical sciences; the official journal of Isfahan University of medical sciences”. Oct; 17(10): 934-937.

20. Girisha KM, et al.2006,'Immunological response to two hepatitis B vaccines administered in two different schedules". Indian Journal of Pediatrics. 2006 Jun;73(6):489-91.

21. Wolfgang Jilg, 1989. "Vaccination against Hepatitis B: Comparison of Three Different Vaccination Schedules". by The University of Chicago..

22. Gallagher KE, 2016. "Factors influencing completion of multi-dose vaccine schedules in adolescents: a systematic review". BMC Public Health. Feb 19;16:172.

23. Qawasmi M, 2015. "Age-dependent decrease of anti-HBs titers and effect of booster doses using 2 different vaccines in Palestinian children vaccinated in early childhood". Human Vaccines Immunotherapeutics.;11(7):1717-24.

24. Fan W, 2016. "Hepatitis B vaccine response in obesity: A meta-analysis". Vaccine Sep 14;34(40):4835-41. 
25. van Wijk PT, 2012. "The risk of blood exposure incidents in dental practices in the Netherlands. Community Dent Oral Epidemiol.” 2012;40:567-73.

26. Averhoff F, 1998. "Immunogenicity of hepatitis B Vaccines. Implications for persons at occupational risk of hepatitis B virus infection”. Am J Prev Med. 1998; 15:1-8.

27. Shaw FE.1989 "Effect of anatomic injection site, age and smoking on the immune response to hepatitis B vaccination".Vaccine; 7:425-30.

28. Weber DJ,1985. "Obesity as a predictor of poor antibody response to hepatitis B plasma vaccine". JAMA; 254:3187-9.

29. Hennig BJ, 2008. "Host genetic factors and vaccine-induced immunity to hepatitis B virus infection". PLoS One; 3:e1898.

30. Honorati MC, 1999. "A mathematical model predicting antihepatitis B virus surface antigen (HBs) decay after vaccination against hepatitis B”. Clin Exp Immunol;116:121-6.

31. Advisory Committee on Immunization Practices; Centers for Disease Control and Prevention CDC.2011, Immunization of health-care personnel: recommendations of the Advisory Committee on Immunization Practices (ACIP) MMWR Recomm Rep;60:1-45. 